\title{
Compound nerve action potential conduction changes in nerve trunk due to tension at various strains and strain rates
}

Shanshan Pu

Chongqing University of Technology

Wei Xie

Sichuan Huada Centre of Forensic Science \& Institute

Hao Wen

Sichuan Huada Centre of Forensic Science \& Institute

Junhong Xiang

Chongqing University of Technology

Xiuju Yang

Chongqing University

Jiang Luo

Sichuan Huada Centre of Forensic Science \& Institute

QianYing Fu

Chongqing University of Technology

Zhixin Pan

Chongqing University of Technology

Chunxiao Lian

Chongqing University of Technology

JingJing Shi

Chongqing University of Technology

Jie Chen

Sichuan Huada Centre of Forensic Science \& Institute

Shengxiong Liu (D89484883@qq.com )

Chongqing University of Technology

\section{Research Article}

Keywords: Compound nerve action potential, Rapid stretch injury, Strain, Strain rates

Posted Date: March 11th, 2021

DOI: https://doi.org/10.21203/rs.3.rs-263548/v1 
License: (c) (i) This work is licensed under a Creative Commons Attribution 4.0 International License. Read Full License 


\title{
Compound nerve action potential conduction changes in
}

\section{nerve trunk due to tension at various strains and strain rates}

\author{
Shanshan Pu ${ }^{\mathrm{a}, \#}$, Wei Xie ${ }^{\mathrm{b}, \#}$, Hao Wen ${ }^{\mathrm{b}, \#}$, Junhong Xiang, ${ }^{\mathrm{a}, \#}$, Xiuju Yang ${ }^{\mathrm{c}}$, Jiang Luo ${ }^{\mathrm{a}, \mathrm{b}}$, \\ QianYing Fu' ${ }^{\mathrm{a}}$, Zhixin Pan ${ }^{\mathrm{a}}$, Chunxiao Lian ${ }^{\mathrm{a}}$, JingJing Shi ${ }^{\mathrm{a}}$, Jie Chen ${ }^{\mathrm{b}, *}$, Shengxiong \\ Liu $^{\mathrm{a}, *}$

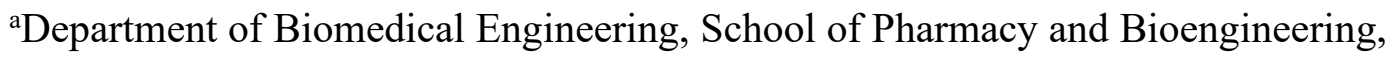 \\ Chongqing University of Technology, Chongqing China \\ ${ }^{\mathrm{b} S i c h u a n}$ Huada Centre of Forensic Science \& Institute \\ ${ }^{\mathrm{c} C}$ College of Bioengineering, Chongqing University, Chongqing, China
}

\# These authors contributed to the work equally and should be regarded as co-first authors: Shanshan Pu, Wei Xie, Hao Wen, Junhong Xiang

* corresponding author: Jie Chen, Shengxiong Liu

Shanshan Pu: 1353304805@qq.com

Wei Xie: 332109970@qq.com

Hao Wen: 820278102@qq.com

Junhong Xiang: 1403568334@qq.com

Xiuju Yang: 530250681@qq.com

Jiang Luo: 1005679544@qq.com

QianYing Fu:1194651879@qq.com

Zhixin Pan:295580219@qq.com

Chunxiao Lian: 330398729@qq.com

JingJing Shi:191827855@qq.com

Jie Chen: 1378782895@qq.com

Shengxiong Liu: 89484883@qq.com 
Abstract : This study investigated compound nerve action potential (CNAP) changes of nerve trunk subjected to stretch at various strains and strain rates. An animal model of rapid stretch injury to nerve based on traffic accidents was developed in order to study CNAP changes of nerve trunk subjected to acute stretch. 18 bullfrogs were dissected and the sciatic nerves' trunks were obtained. The nerve trunk was stretched by $6 \%, 12 \%$ or $18 \%$ of its length and the tensile strain rates were $30 \mathrm{~s}-1$ and 50s- 1 respectively. The results showed that at $6 \%$ strain and $30 \mathrm{~s}-1$ strain rate, the amplitude, action potential duration and conduction velocity of CNAP had a slight change. When the strain and strain rate continued to be increased, the amplitude, conduction velocity and area under the curve would be decreased while the action potential duration would be increased. At $18 \%$ strain and $50 \mathrm{~s}^{-1}$ strain rate, CNAP conduction was almost completely blocked. The different strains or strain rates had a significant effect on nerve signal conduction. Under the same strain, a higher strain rate would increase the probability of nerve conduction block. These findings are of great significance to study the injury mechanism of rapid nerve stretch and to evaluate the nerve functional damage level in traffic accidents.

Keywords: Compound nerve action potential, Rapid stretch injury, Strain, Strain rates

\section{Introduction}

The Abbreviated Injury Scale (AIS) is commonly used in the damage evaluation of traffic accidents ${ }^{[1]}$. However, most of the rating indicators in AIS are morphological and qualitative parameters, such as fractures, bleed, skin abrasions, and tissue contusion. In fact, central nervous system injury and peripheral nervous system injury are the most common injuries and are also the primary causes of death in traffic accidents. Functional injury of nervous system may not only cause early abnormality of related physiological activities, but also lead to permanent physiological dysfunction. Some studies showed that the collision process would cause a strain of $6 \%$ or even more in the brain tissue ${ }^{[2]}$. The finite element simulation results showed that the angular velocity of brain increased from 0 to $70 \mathrm{rad} / \mathrm{s}$ within $10 \mathrm{~ms}$ and then decreased to $0 \mathrm{rad} / \mathrm{s}$ within $20 \mathrm{~ms}$, which could cause $14.8 \%$ shear strain in the brain tissue ${ }^{[3]}$. Some researches studied traumatic brain injury(TBI) and traumatic axonal injury(TAI) through strain, strain rate and production of strain and strain rate(PSSR), and established the relationship between PSSR and injury probability of brain tissue ${ }^{[4]}$. While most of the above studies were based on simulation models or dummy models which objectively had physiological limitations. In traffic accidents, because of the short duration and high intensity of the impact process, nerves often experienced large strains and strain rates.

The effect of nerve stretch on nerve function may be two-sided. Safe nerve stretching can be therapeutic for some conditions. Due to the differences between the published research results, it is impossible to draw precise conclusions of the safety limits of nerve stretching ${ }^{[5]}$. The nerve structure and the injury degree of nerve function could be analyzed by measuring CNAP. Quasi-static stretching or low-speed stretching is the mainstream of nerve stretching research ${ }^{[6-9]}$. Back in 1992, Wall established animal models of quasi-static nerve stretch injury and found that the amplitude of CNAP had decreased by $70 \%$ after $6 \%$ tensile strain at one hour and conduction was completely blocked after $18 \%$ tensile strain ${ }^{[7]}$. The amplitude and area under the curve of CNAP are correlated with nerve fiber numbers ${ }^{[10,11]}$. The action potential duration is associated with synchronous excitement extent of nerve fiber. The lower synchronous 
excitement extent of nerve fiber is, the lower amplitude is, and the wider action potential duration is ${ }^{[12]}$. The nerve conduction velocity can reflect the integrity of myelin sheath ${ }^{[13]}$. The amplitude, action potential duration and area under the curve will change significantly when nerve trunk is diseased or damaged ${ }^{[14,15]}$. Other studies showed that the amplitude and area under the curve of CNAP decreased with the increasing of strain ${ }^{[9]}$. The previous experiments identified a $6 \%-$ $100 \%$ range of strain values for conduction block ${ }^{[6]}$. But in these experiments these nerves had been stretched and maintained for a long time which would result in additional injury and difference to the real traffic injury.

So far, there is still a lack of research on rapid nerve stretch injury especially the functional injury which caused by vehicle collision or sporting accidents. In this paper, an animal model of rapid nerve stretch injury was developed, where different strains and strain rates were used to stretch nerves, and the changes of action potential conductivity of nerve trunk were explored. It will provide a new idea and experimental supports for the mechanism research of the traffic nerve injury and the evaluation of functional injury degree.

\section{Materials and methods}

\subsection{Study Animals}

Eighteen bullfrogs $(100 \sim 150 \mathrm{~g})$ were purchased from Liaoning Changsheng Biotechnology Co., Ltd. Try to reduce the number of experimental animals and reduce the pain of animals. All studies and procedures were approved by the academic and ethical committee of Chongqing University of Technology(permit number 202109). The study was carried out in compliance with the ARRIVE guidelines. All procedures undertaken in this study were in accordance with approved guidelines.

2.2 Neurophysiological measurement

CNAP was recorded by biomedical signal acquisition system. The signal sampling frequency was $20000 \mathrm{~Hz}$, stimulating frequency was $5 \mathrm{~Hz}$, stimulus square wave duration was $0.1 \mathrm{~ms}$, and the low-pass filter frequency was $1000 \mathrm{~Hz}$.

\subsection{Statistical analysis}

Spss26.0 was used for data analysis and the data were presented as mean \pm SEM. One-way ANOVA with Least-Significant Different test was used for comparisons of amplitude, action potential duration and area under the curve between different experimental groups. A p-value of less than 0.05 was considered to be statistically significant.

\subsection{Experimental methods}

First, the nerve trunk was placed on the CNAP measuring device, S1 and S2 were used to stimulate nerve trunk and CNAP was recorded by R1 and R2 (Figure 1). Then, it was removed from the CNAP recording device and fixed on the nerve stretching device. The strain and strain rate of the nerve were regulated by the height of the loading slider and the blocking slider (Figure 2). Finally, after the nerve was stretched rapidly, the nerve trunk was stimulated with the same voltage and the CNAP was recorded again.

Figure 1 The approaches of compound nerve action potential recording (S1 and S2 are stimulating electrodes, R1 and R2 are measuring electrodes)

Figure 2 Nerve stretch model

\section{Results}


In general, the CNAP was significantly different between each of the six groups (Table 1, $P<0.05$ ). In the $6 \%$ strain and $30 \mathrm{~s}^{-1}$ group, nerve amplitude increased $1.5 \%$ on average and the area under the curve increased $2.1 \%$ on average. With the increasing of strain and strain rate, the amplitude, nerve conduction velocity and area under the curve decreased significantly while the action potential duration increased significantly (Figure $3,4,5,6 ; P<0.05$ ). In the $18 \%$ strain and $50 \mathrm{~s}^{-1}$ group, conduction was almost completely blocked.

Besides, the strain and strain rate of nerve stretch had great influence on nerve signal conduction. A higher strain rate at the same strain would also have a significant effect on signal transmission $(P<0.05)$.

Table 1 Effects of different strain and strain rate on compound nerve action potential

Figure 3 Changes of amplitude before and after nerve stretching

Figure 4 Changes of action potential duration before and after nerve stretching

Figure 5 Changes of nerve conduction velocity reduction before and after nerve stretching

Figure 6 Changes of area under the curve before and after nerve stretching

It could be seen from this $3 \mathrm{D}$ scatter plot that both strain and strain rate had a significant effect on the amplitude of nerve signal(Figure 7). SPSS26.0 software was used for linear regression analysis. It was found that there were highly significant positive correlations between amplitude reduction ratio $(\mathrm{y})$ and strain $\left(\mathrm{x}_{1}\right)$ or strain rate $\left(\mathrm{x}_{2}\right)$. The linear correlation coefficient $\mathrm{R}$ was 0.977 and the linear correlation coefficient $\mathrm{R}^{2}$ was 0.955 , which showed that there was an obvious linear correlation. The linear regression equation was $\mathrm{y}=614.734 \mathrm{x}_{1}+1.112 \mathrm{x}_{2}-75.729$. The $\mathrm{x}_{1}$ coefficient ranged from 564.733 to 664.735 . The $\mathrm{x}_{2}$ coefficient ranged from 50.866 to 1.359. The equation constant ranged from -87.277 to -64.182 (Table 2).

Figure 7 The influence of strain and strain rate on amplitude

Table 2 Slope and intercept range of linear regression statistics

\section{Discussion}

Neuroelectrophysiology is an important method to explore nerve's function, with long history of research ${ }^{[16,17]}$. The CNAP is the total potential produced by various nerve fibers as the nerve trunk is stimulated ${ }^{[18]}$. CNAP recording, a classic and mature electrophysiological method, is a useful tool for assessing peripheral nerve diseases and nerve repair. CNAP has a unique diagnostic value for the analysis of nerve function and structure ${ }^{[19,20]}$.

Many studies have shown that stretch could destroy myelin ${ }^{[21,22]}$ and cytoskeleton ${ }^{[23,24]}$. Some scholars ${ }^{[9]}$ stretched the nerve with different strains $(<10 \%, 10-20 \%$, and $>20 \%)$ and strain rates $(0.01 \mathrm{~mm} / \mathrm{sec}, 1 \mathrm{~mm} / \mathrm{sec}$, or $15 \mathrm{~mm} / \mathrm{sec})$, the maximum strain rate is $0.3 \mathrm{~s}^{-1}$, which is lower than the nerves strain rate in traffic accidents. So the electrophysiological characteristics of these injuries should be quite different from the electrophysiological characteristics of acute injuries. In this study, a model of rapid nerve injury was built and the changes of CNAP were measured to evaluate the effects of different strains and strain rates on nerve conduction.

This study has shown that the amplitude, area under the curve and action potential duration rose slightly and the nerve conduction velocity changed little at $6 \%$ strain and $30 \mathrm{~s}^{-1}$ strain rate. This finding, from the perspective of neuroelectrophysiology, explained the view that nerve stretch within the safety limit may be beneficial to neurotherapy. With the increase of strain and strain rate, the amplitude nerve conduction velocity and area under the curve of the CNAP continued to decrease, but action potential duration increased significantly. The decrease of amplitude meant that the number of nerve fibers that could generate action potential was reduced. 
The increase of action potential duration and the decrease of conduction velocity indicated that the synchronous excitability of nerve fibers decreased and the myelin sheath of nerve fiber was exfoliated. At $18 \%$ strain and $50 \mathrm{~s}^{-1}$, nerve conduction was completely blocked meaning the loss of nerve function.

Previous studies have not focused on the effect of high strain rate on nerve stretch injury. The study in this paper has shown that under the same strain condition higher strain rate would lead to lower amplitude, conduction velocity and area under the curve, and longer action potential duration. That was to say higher strain rates will reduce the strain threshold of nerve injury. This study investigated the changes of CNAP conduction characteristics after rapid stretch injury to nerve and obtained the conduction block threshold of nerve signal under different strains and strain rates stretching. The results further showed that there existed a linear relation between amplitude reduction ratio and strain or strain rate. With the increase of PSSR, nerve signal conduction was affected, nerve conduction was completely blocked when PSSR was 9(Figure 8). It is helpful to quantitatively analyze the effect of different strains or strain rates on nerve rapid stretching and to establish the level of nerve functional injury in traffic accidents or sport accidents. This work provided ideas and data for the evaluation and research of nerve functional injury under rapid stretch.

\section{Conflicts of interest}

Figure 8 The relationship between nerve signal conduction and PSSR

None declared.

\section{Acknowledgments}

This paper was supported by the National Natural Science Foundation of China (31200709)

\section{References}

[1] Thomas A. Gennarelli, Elaine Wodzin. AIS 2005 : A contemporary injury scale. 2006, 37(12):1083-1091.

[2] Libing Chen,Jianbao Han.Simulating calculation of passenger brain tissue deformation during vehicle crash accident using $f$ inite element method[J].Chinese Journal of Trauma,2003, 19(009):521-523.

[3] Shongxiong Liu,Zhiyong Yin,HuiZhao,et al.Research on brain tissue mechanical style in brain model deceleration impact[J].Journal of Medical Biomechanics,2009(06):458-461.

[4] Runzhou Zhou. DEVELOPMENT OF RAT HEAD FINITE ELEMENT MODEL AND TISSUE LEVEL BIOMECHANICAL THRESHOLD FOR TRAUMATIC AXONAL INJURY. [D]. Detroit Michigan: Wayne State University, 2020.

[5] Mahan Mark A. Nerve stretching: a history of tension.. 2019, 132(1):252-259.

[6] Wall E J, Massie J B, Kwan M K, et al. Experimental stretch neuropathy. Changes in nerve conduction under tension.. 1992, 74(1):126-9.

[7] Anita Singh, Ying Lu, Chaoyang Chen, et al. Mechanical properties of spinal nerve roots subjected to tension at different strain rates. 2005, 39(9):1669-1676.

[8] Singh Anita, Kallakuri Srinivasu, Chen Chaoyang, et al. Structural and functional changes in nerve roots due to tension at various strains and strain rates: an in-vivo study.. 2009, 26(4):627-40.

[9] Mark Stecker, Kelly Baylor, Jacob Wolfe, et al. Acute nerve stretch and the compound motor action potential. 2011, 6(1):e11-e22. 
[10] Huan Wang, Eric J. Sorenson, Robert J. Spinner, et al. Electrophysiologic findings and grip strength after nerve injuries in the rat forelimb. 2008, 38(4):1254-1265.

[11] Yongping Li,Jie Lao,Xin Zhao,et al.The correlation between compound nerve action potential and the number of the regenerated nerve fibers[J].Chinese Journal of Hand Surgery,2012(02):102-106.

[12] Jian L , Fang C , Jia-Xiang X . Analysis and discussion of compound action potentials with nerve stem[J]. Sichuan Journal of Physiological Sciences, 2008(03):103-105.

[13] Kane N M , Oware A . Nerve conduction and electromyography studies[J]. Journal of Neurology, 2012, 259(7):1502-1508.

[14] G. H. Vliet, J. Holsheimer, D. Bingmann. Calculation of the conduction velocity of short nerve fibres. 1980, 18(6):749-757.

[15] Li Jianming, Shi Riyi. Stretch-induced nerve conduction deficits in guinea pig ex vivo nerve.. 2007, 40(3):569-78.

[16] Saeed M A, Gatens P F. Compound nerve action potentials of the medial and lateral plantar nerves through the tarsal tunnel.. 1982, 63(7):304-7.

[17] Lang A H,Puusa A. Dual influence of temperature on compound nerve action potential.[J]. Journal of the neurological sciences, 1981,51(1):81-88.

[18] Yongping Li, Jie Lao, Xin Zhao, et al. The optimal distance between two electrode tips during recording of compound nerve action potentials in the rat median nerve. 2014, 9(2):171-178.

[19] Kim Daniel H, Murovic Judith A, Kim Yong-Yeon, et al. Surgical treatment and outcomes in 45 cases of posterior interosseous nerve entrapments and injuries.. 2006, 104(5):766-77.

[20] Kim Daniel H, Murovic Judith A, Kim Yong-Yeon, et al. Surgical treatment and outcomes in 15 patients with anterior interosseous nerve entrapments and injuries.. 2006, 104(5):757-65.

[21] Ichiro Abe, Akihito Tsujino, Yuki Hara, et al. Paranodal demyelination by gradual nerve stretch can be repaired by elongation of internodes. 2002, 104(5):505-512.

[22] Haftek J. Stretch injury of peripheral nerve. Acute effects of stretching on rabbit nerve.. 1970, 52(2):354-65.

[23] Jafari S S, Nielson M, Graham D I, et al. Axonal cytoskeletal changes after nondisruptive axonal injury. II. Intermediate sized axons.. 1998, 15(11):955-66.

[24] Maxwell W L, Graham D I. Loss of axonal microtubules and neurofilaments after stretch-injury to guinea pig optic nerve fibers.. 1997, 14(9):603-14. 
Figures

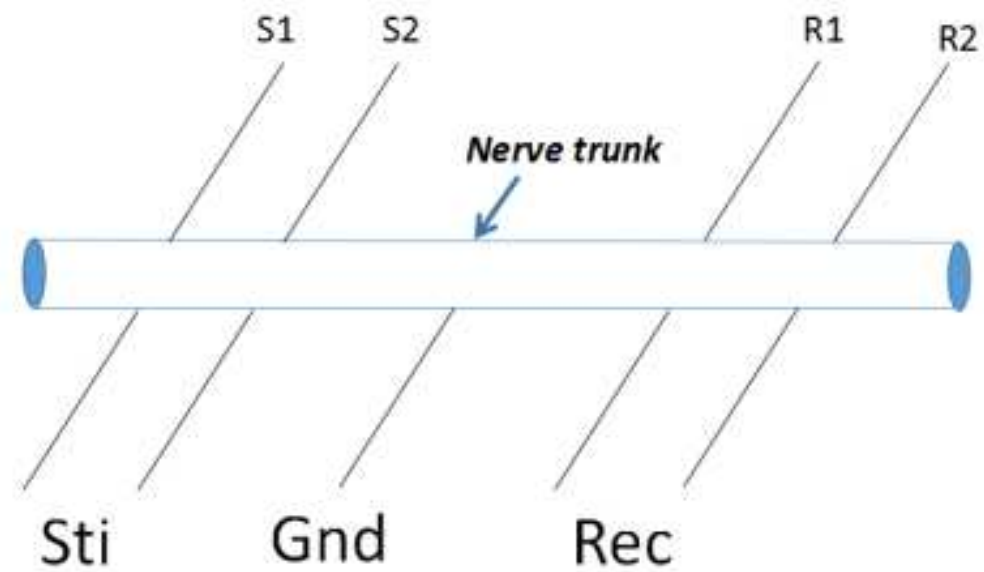

\section{Figure 1}

The approaches of compound nerve action potential recording (S1 and S2 are stimulating electrodes, R1 and R2 are measuring electrodes)

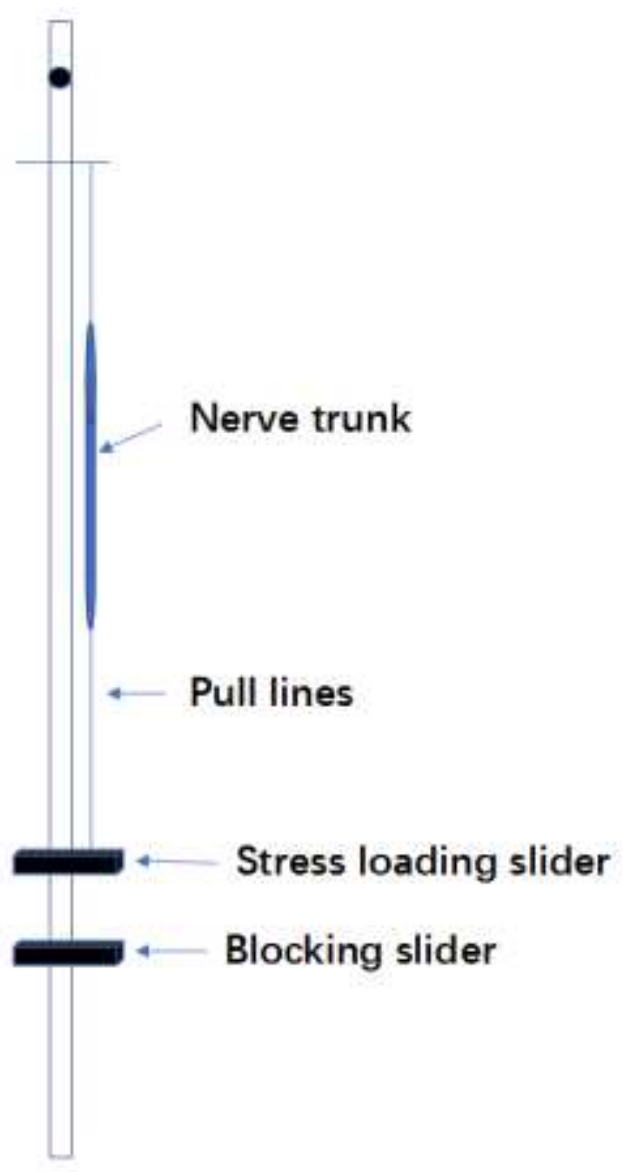

Figure 2

Nerve stretch model 


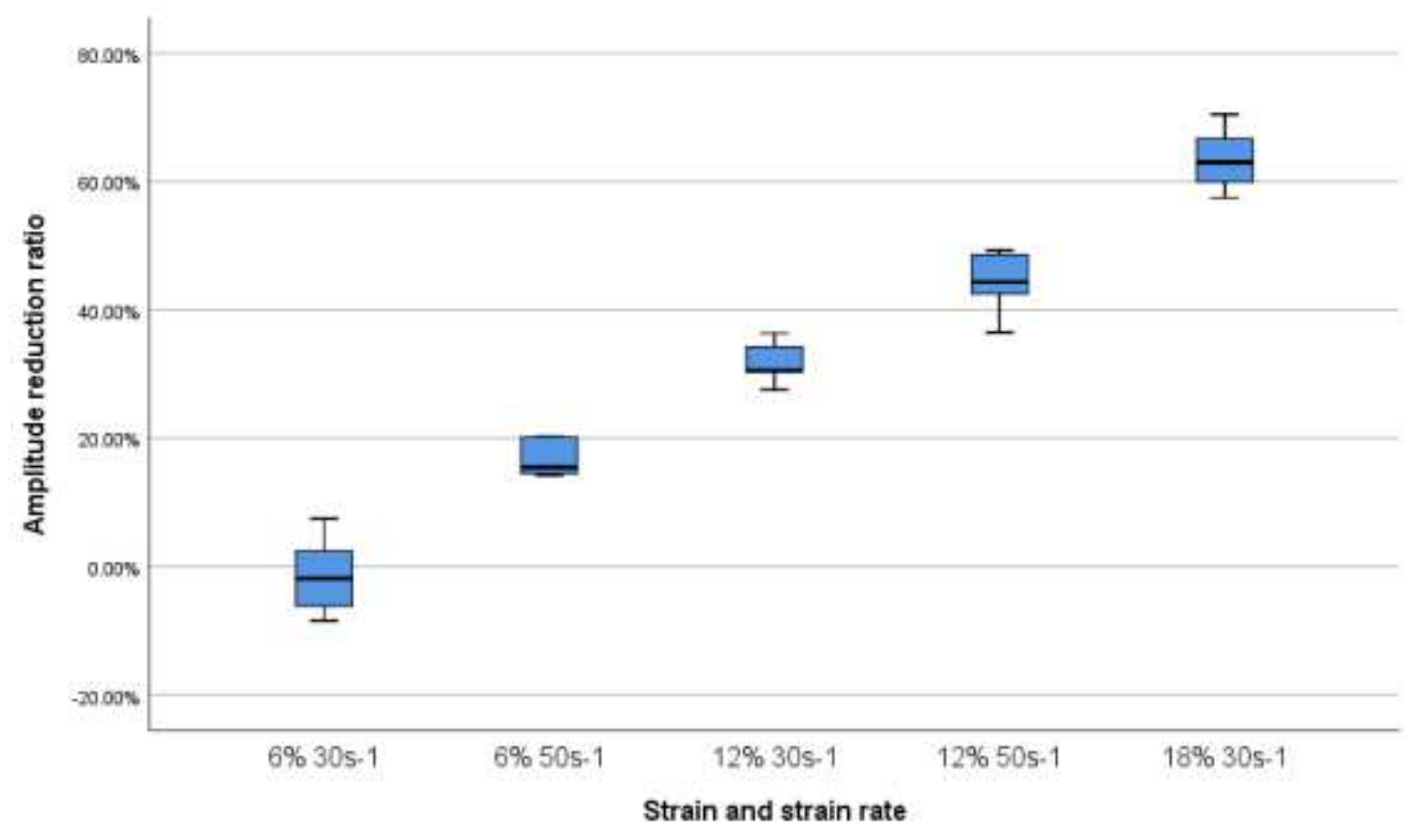

Figure 3

Changes of amplitude before and after nerve stretching

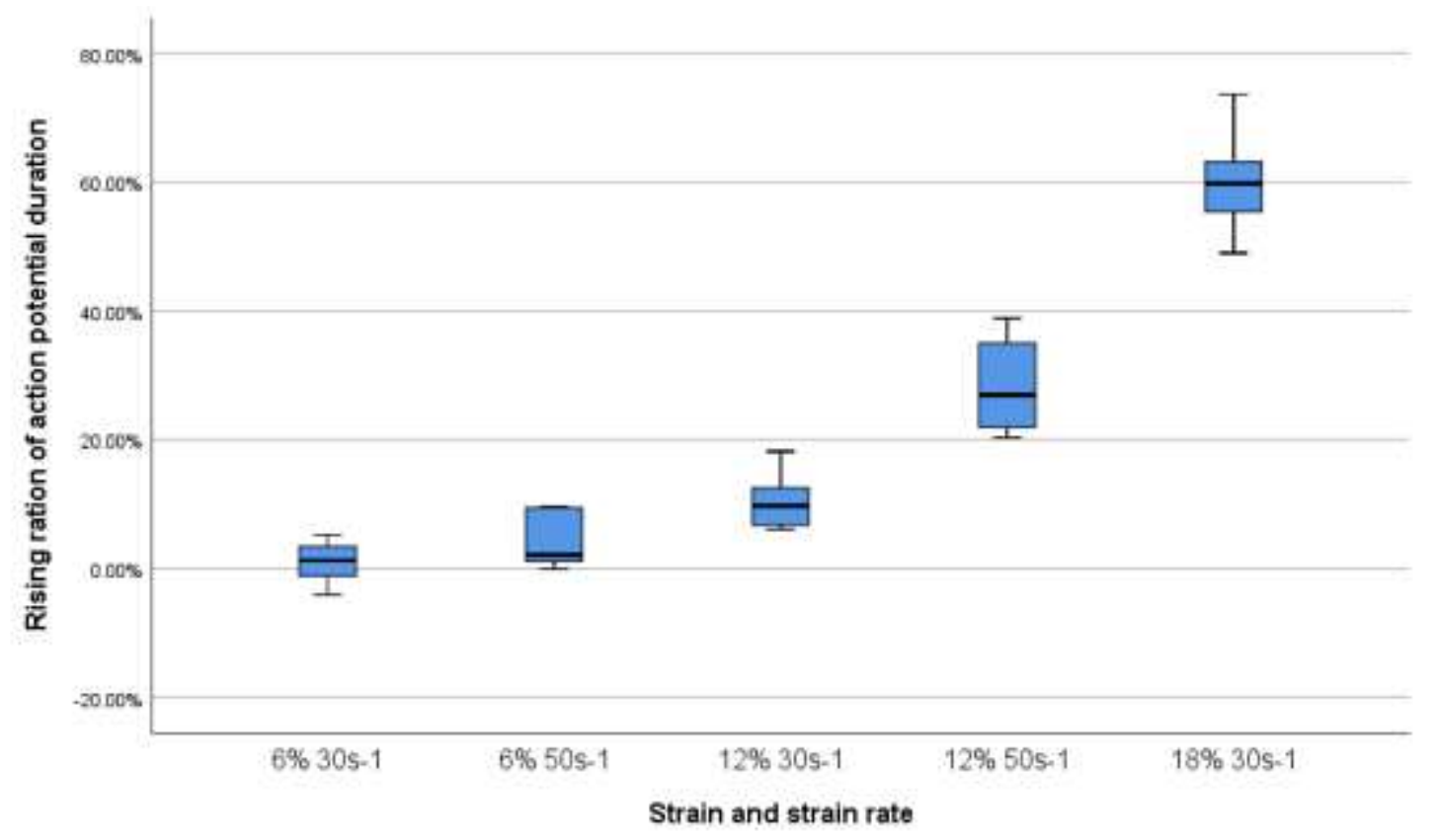

Figure 4

Changes of action potential duration before and after nerve stretching 


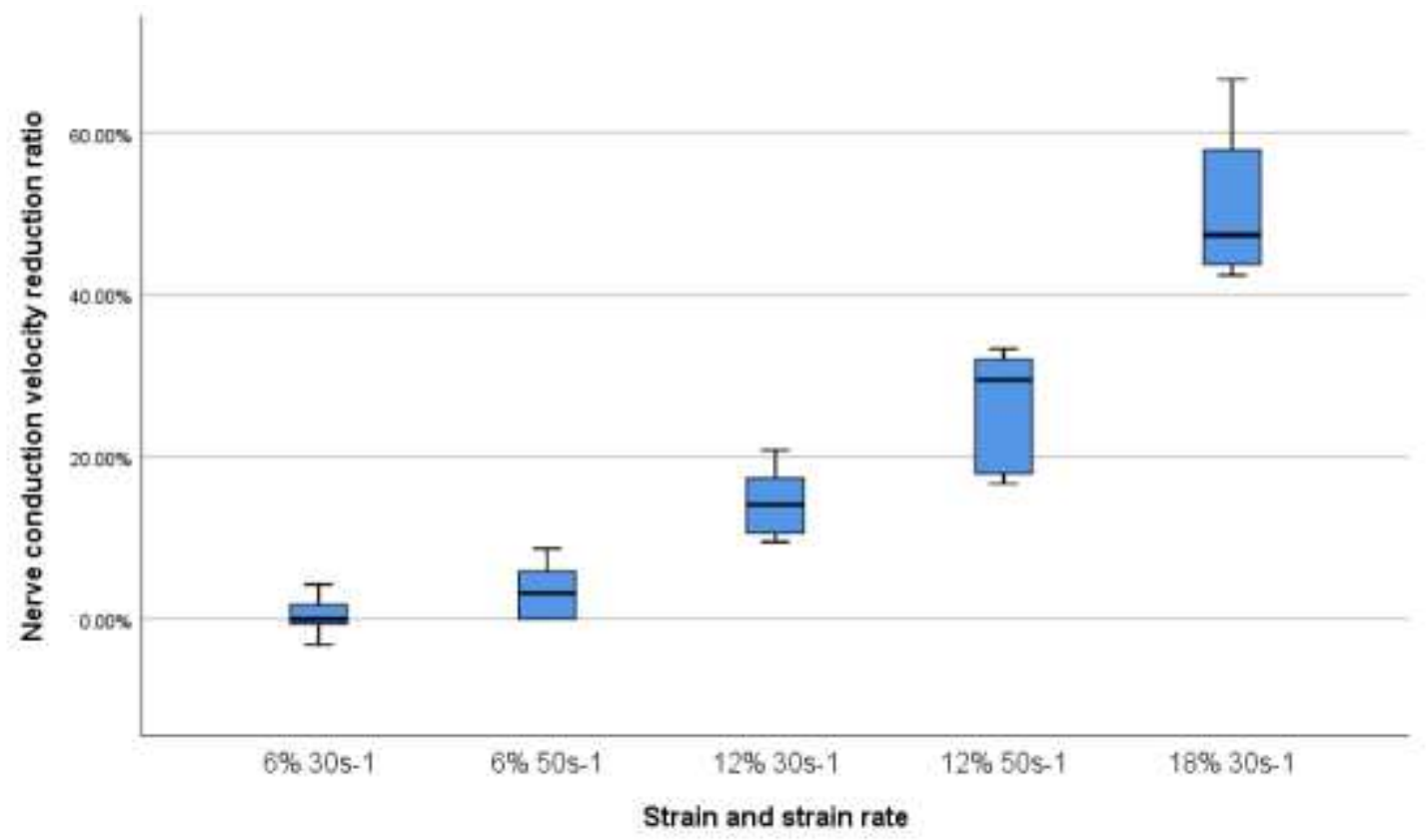

Figure 5

Changes of nerve conduction velocity reduction before and after nerve stretching

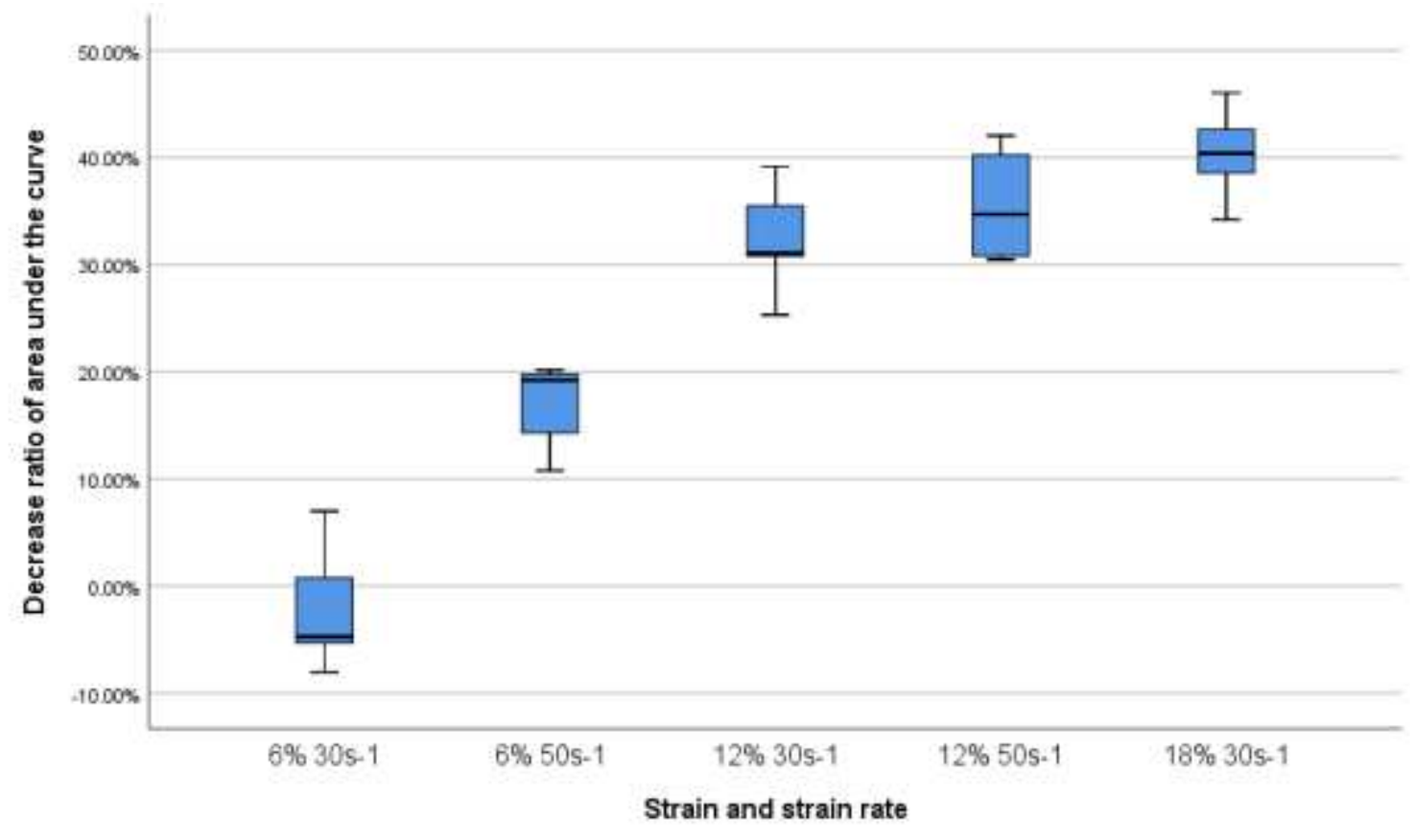

Figure 6

Changes of area under the curve before and after nerve stretching 


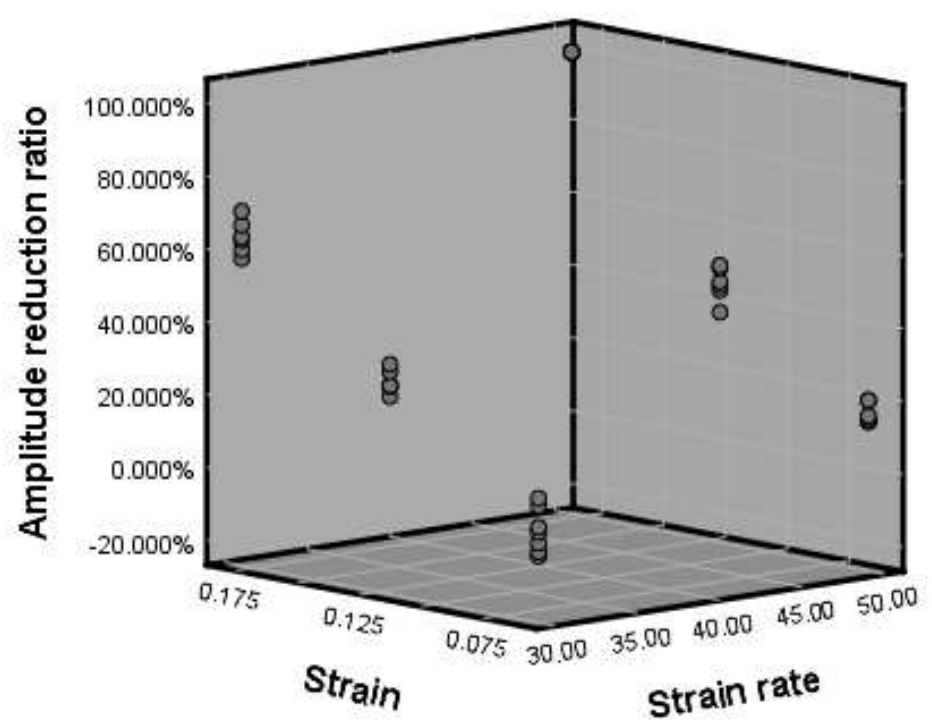

Figure 7

The influence of strain and strain rate on amplitude 


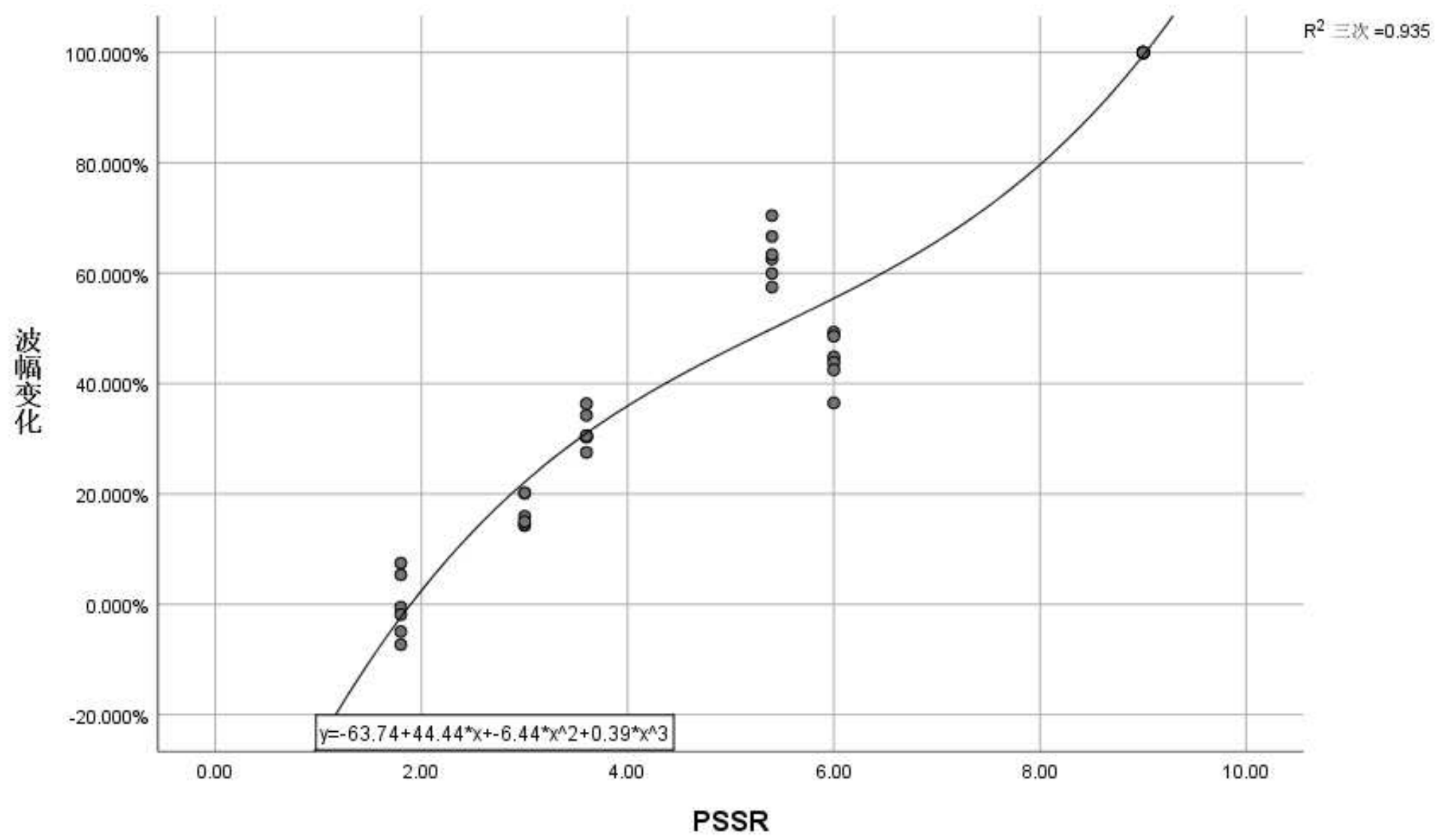

Figure 8

The relationship between nerve signal conduction and PSSR 